\title{
Mortality Risk and the Value of a Statistical Life: The Dead-Anyway Effect Revis(it)ed
}

\author{
FRIEDRICH BREYER \\ Department of Economics, University of Konstanz and DIW Berlin \\ STEFAN FELDER \\ Faculties of Medicine and Economics, University of Magdeburg
}

Received December 15, 2003; Revised July 7, 2004

\begin{abstract}
In the expected-utility theory of the monetary value of a statistical life, a well-known result found by Pratt and Zeckhauser [1996] asserts that an individuals' willingness to pay (WTP) for a marginal reduction in mortality risk increases with the initial level of risk. Their reasoning is based on the so-called "dead-anyway effect" which states that marginal utility of a dollar in the state of death is smaller than in the state of survival. However, this explanation is based on the absence of markets for contingent claims, i.e. annuities and life insurance. This paper reexamines the relationship between WTP and the level of risk under more general circumstances and establishes two main results: first, when insurance markets are perfect, for a risk-averse individual without a bequest motive, marginal WTP for survival does increase with the level of risk but this occurs for a different reason, namely an income effect. Secondly, when the individual has a bequest motive and is endowed with a sufficient amount of wealth from human capital, the effect of initial risk on WTP for survival is reversed: the higher initial risk the lower the value of a statistical life. In the imperfect-markets case we interpret this result as a "constrained-bequest effect".
\end{abstract}

Key words: value of life, expected utility, willingness to pay, insurance markets

JEL Classification No.: $\quad$ D8, H43, I18

\section{Introduction}

The expected-utility foundation of the (monetary) value of a statistical life is based on the seminal contributions by Jones-Lee [1976, chapter 5] and Rosen [1988] or, more recently, Johansson [1996, 2002]. A key question in this literature is how this value, i.e. the willingness to pay (WTP) for small increments in the chance of survival is influenced by the characteristics of the person under consideration, in particular by his (bequeathable or non-bequeathable) wealth and the initial value of the mortality risk. This question is of significant policy relevance because in most practical applications, marginal WTP of the exposed persons can not be directly observed but must be inferred from e.g., questionnaire responses of a different population, which is often much less exposed. If there is a systematic 
relationship between WTP and the level of risk, then such an inference may be unwarranted if the results are not appropriately corrected.

The current state of knowledge on this question is based on a paper by Pratt and Zeckhauser [1996] who claim that an individual's WTP will be monotonically increasing in the initial level of risk due to a "dead-anyway" effect (henceforth: DAE). ${ }^{1}$ This means that if marginal utility of a dollar when dead (or marginal utility of bequest) is lower than marginal utility of a dollar when alive (or marginal utility of consumption), then increasing the mortality risk reduces the expected marginal utility of a dollar and, thus, increases WTP, defined as the marginal rate of substitution between the chance of survival and wealth.

However, this explanation may be simplistic because it is explicitly based on the complete absence of contingent-claims markets (i.e. annuities and life insurance) ${ }^{2}$ where the individual can trade wealth across different states of nature and thus equalize marginal utility of wealth. It is a priori unclear whether this result will hold in a world of perfect contingent-claims markets. The present paper is devoted to a thorough theoretical analysis of the determinants of WTP for small increments in the probability of survival under various circumstances. The main question to be answered is what set of assumptions is required for the positive effect of the level of risk to hold, and under what reasonable assumptions we might get a different result. Moreover, we shall examine whether the intuitive explanation given by Pratt and Zeckhauser with reference to the DAE always applies.

The paper is organized as follows. Section 2 introduces the basic model without a bequest motive and distinguishes between three organization of the insurance market: perfect, imperfect and non-existent. Section 3 adds bequests and Section 4 summarizes the findings and compares them to the available empirical evidence.

\section{The model without a bequest motive}

\subsection{The general case}

We consider an individual whose utility $u(c)$ is a positive and strictly increasing function of her consumption $c$ provided she stays alive, i.e. $u>0, u^{\prime}>0$. With zero utility (and zero marginal utility of consumption) assigned to the state of death, the expected utility of the individual is

$$
E U=\pi \cdot u(c)
$$

where $\pi$ is the probability of survival. The individual's budget is composed of survival income $H$, which is lost upon death, and bequeathable wealth $V$. Survival income includes human capital and annuities that already exist at the time the individual reveals her willingness to pay for an increase in survival probability. In the insurance market, $p(\pi)$ is the price of the annuity which will depend on the organization of the market. On a perfect market, the survivors are offered fair tontine shares, i.e. $p(\pi)=\pi, p^{\prime}(\pi)=1$ and $p^{\prime \prime}(\pi)=0$, see Rosen, [1988]. ${ }^{3}$ The other extreme is a non-existent insurance market where $p(\pi)=1, p^{\prime}(\pi)=$ 0 and $p^{\prime \prime}(\pi)=0$. In the general case, $p(\pi) \geq \pi, p^{\prime}(\pi) \geq 0$, while $p^{\prime \prime}(\pi)$ can have any sign. 
Individuals then will trade their total non-human wealth $V$ for a contract which pays $V / p(\pi)$ in case of survival. Thus, the surviving individual faces the following budget constraint

$$
c=V / p(\pi)+H,
$$

giving rise to the expected indirect utility function

$$
E U(\pi, V, H)=\pi \cdot u(H+V / p(\pi)) .
$$

The marginal willingness to pay $d V$ for a small increase in survival probability $d \pi$ equals the marginal rate of substitution between survival and bequeathable wealth

$$
m(\pi, V, H)=-\left.\frac{d V}{d \pi}\right|_{d E U=0}=\frac{\frac{\partial E U}{\partial \pi}}{\frac{\partial E U}{\partial V}}=\frac{u-\pi \cdot u^{\prime} \cdot V \cdot p^{\prime} / p^{2}}{u^{\prime} \cdot \pi / p}=\frac{p}{\pi} \cdot \frac{u}{u^{\prime}}-p^{\prime} \cdot \frac{V}{p} .
$$

The value of life consists of two parts. ${ }^{4}$ First, the change in survival probability directly affects expected utility by $u \cdot d \pi$, or by $\left[u /\left(u^{\prime} \cdot \pi / p\right)\right] \cdot d \pi$ in monetary terms. The second part comes from the income effect through the change in the annuity price captured by the budget constraint (2).

Taking into account the budget constraint has the following implication: The individual revealing her willingness to pay for an increase in survival probability assumes that the terms of her insurance contract will be adjusted correspondingly. This is certainly an unrealistic assumption with respect to WTP data from surveys. On the other hand, it is the correct procedure when the data are used to inform public policy because the budget constraint is valid at the society level. On this distinction, see also Johansson [2002], Section 4. When the income effect through the individual's net position on the insurance market is not considered, then the change in survival probability only has a direct effect on expected utility.

The derivative of $m(\pi, V, H)$ with respect to $\pi$ gives

$$
\begin{aligned}
\frac{\partial m(\pi, V, H)}{\partial \pi}=-\left.\frac{\partial^{2} V}{\partial \pi^{2}}\right|_{d E U=0} \\
=\frac{\left[p^{\prime} \cdot u-p \cdot u^{\prime} \cdot\left(V \cdot p^{\prime} / p^{2}\right)\right] \cdot \pi \cdot u^{\prime}-\left[u^{\prime}-\pi \cdot u^{\prime \prime} \cdot\left(V \cdot p^{\prime} / p^{2}\right)\right] \cdot p \cdot u}{\pi^{2} \cdot u^{\prime 2}} \\
\quad+\frac{V}{p} \cdot\left[\frac{p^{\prime 2}}{p}-p^{\prime \prime}\right] \\
=-\frac{u}{\pi \cdot u^{\prime}}\left(\frac{p}{\pi}-p^{\prime}\right)-p^{\prime} \cdot\left[-\frac{u^{\prime \prime} \cdot u}{\pi \cdot u^{\prime 2}}+\frac{1}{p} \cdot\left(\frac{p}{\pi}-p^{\prime}\right)+\frac{p^{\prime \prime}}{p^{\prime}}\right] \cdot \frac{V}{p} .
\end{aligned}
$$

Provided that marginal loading does not exceed average loading $p^{\prime} \leq p / \pi$ and the individual is risk averse $\left(u^{\prime \prime}<0\right)$, WTP for survival increases with the initial level of risk. If we assume, 
in particular, that loading is proportional to the expected insurance payment,

$$
p(\pi)=(1+\lambda) \cdot \pi
$$

and thus $p^{\prime}=1+\lambda, p^{\prime \prime}=0$, we get from (5)

$$
\frac{\partial m}{\partial \pi}=\frac{u^{\prime \prime} \cdot u}{u^{\prime 2}} \cdot \frac{V}{\pi^{2}}<0 .
$$

The change in WTP from a decrease in the mortality comes through the income effect on the insurance market: decreasing the survival probability $\pi$ will decrease the annuity price, and increase the individual's income. This, in turn, with a concave utility function lowers the marginal utility of wealth, which means that more wealth will be sacrificed for a given increase in the probability of survival.

\subsection{No insurance markets}

With non-existent insurance (case $n i$ ), we obtain

$$
m_{n i}=\frac{u}{u^{\prime} \cdot \pi},
$$

and

$$
\frac{\partial m_{n i}}{\partial \pi}=-\frac{u}{u^{\prime} \cdot \pi^{2}}<0 .
$$

This is the DAE that Pratt and Zeckhauser had in mind. From (8) one sees that in this model the elasticity of the value of life with respect to mortality $(\partial m / m) /(\partial \pi / \pi)$ is unity and does not depend on the level of the initial risk. Marginal utility is not affected by the change in mortality risk as the consumption level in the state of survival remains constant. This effect comes through the objective function: expected marginal utility of consumption falls when mortality risk rises.

The DAE is also present if the price of annuities is not adjusted for a change in survival probability, a situation that might be relevant for surveys where persons are asked for their WTP for survival. Then, we have $m=p /\left(\pi \cdot u^{\prime}\right)$ and $\partial m / \partial \pi=-p \cdot u /\left(u^{\prime} \cdot \pi^{2}\right)$.

\subsection{Perfect insurance markets}

With a perfect insurance market (case pi), (4) and (5) reduce to

$$
m_{p i}=\frac{u}{u^{\prime}}-\frac{V}{\pi}
$$


and

$$
\frac{\partial m_{p i}}{\partial \pi}=\frac{u^{\prime \prime} \cdot u}{u^{\prime 2}} \cdot \frac{V}{\pi^{2}}<0
$$

and we obtain the income effect only. Here, risk aversion is a necessary condition for $\partial m / \partial \pi<0$. It is noteworthy that the change in WTP for survival is the same with perfect markets than with constant loading. In both cases we have $p / \pi=p^{\prime}$ (see (5)), so that the DAE is offset by a price effect (expected marginal utility of income increases with a rise in survival probability, while it decreases with a rise in the price of the annuity). Hence, the change in WTP is determined by the income effect.

This one-period analysis can be extended to a continuous consumption life-cycle model with actuarially fair annuities. Felder, Meier and Schmitt [2000] prove for a permanent change of the mortality risk in the sense of Johansson [1996] the DAE to hold.

\section{Adding bequests}

\subsection{The general case}

We extend the model by assuming that individuals care for their heirs. Expected utility is defined as follows

$$
E U=\pi \cdot u_{L}\left(c_{L}\right)+(1-\pi) \cdot u_{D}\left(c_{D}\right)
$$

where $u_{L}$ is utility of consumption in the state of survival $\left(c_{L}\right)$ and $u_{D}$ represents the bequest motive, i.e. utility derived from the consumption of the heirs in the state of death $\left(c_{D}\right)$. The state dependent utilities are both assumed to be strictly increasing: $u_{L}^{\prime}, u_{D}^{\prime}>0$.

As is common in the pertinent literature (e.g. Jones-Lee [1976]), we assume that individuals prefer survival to death, and that marginal utility when alive is higher than marginal utility when dead at any level of consumption:

$$
\begin{gathered}
u_{L}(c)>u_{D}(c) \quad \forall c, \\
u_{L}^{\prime}(c)>u_{D}^{\prime}(c) \quad \forall c .
\end{gathered}
$$

An insurance market offers $1 / p_{L}(\pi) \$$ (with $p_{L}(\pi) \geq \pi$ ) for any dollar spent on an annuity contract and $1 /\left(1-p_{D}(\pi)\right) \$\left(\right.$ with $\left.1-p_{D}(\pi) \geq 1-\pi\right)$ for any dollar spent on life insurance. This gives rise to the budget constraint

$$
p_{j}(\pi) \cdot c_{L}+\left(1-p_{j}(\pi)\right) \cdot c_{D}=V+p_{j}(\pi) \cdot H, \quad j=L, D
$$

When intended bequests are present, we thus have two insurance markets that can either be perfect, imperfect or non existent. Maximizing (12) subject to (15) leads to the following 
FOC for an interior optimum:

$$
\frac{u_{L}^{\prime}\left(c_{L}\right)}{u_{D}^{\prime}\left(c_{D}\right)} \cdot \frac{\pi}{1-\pi}=\frac{p_{j}}{1-p_{j}} .
$$

A corner solution with $c_{L}=V+H$ and $c_{D}=V$ is optimal whenever at this point

$$
\frac{p_{D}}{1-p_{D}} \leq \frac{\pi}{1-\pi} \cdot \frac{u_{L}^{\prime}(V+H)}{u_{D}^{\prime}(V)} \leq \frac{p_{L}}{1-p_{L}} .
$$

The same corner solution naturally occurs when insurance markets do not exist. In perfect insurance markets, $p_{L}=p_{D}=\pi$, and the individual reallocates her wealth until the marginal utility of a dollar is the same in both states.

The solution is a maximum if the indifference curves for expected utility are globally convex, which requires concave utility functions in both states of nature $\left(u_{D}^{\prime \prime}<0, u_{L}^{\prime \prime}<0\right)$. Convex indifference curves together with (14) imply that $c_{L}-c_{D}>0$ in the optimum. Furthermore, by (13) and the monotonicity of the utility function, utility in the state of survival exceeds utility in the state of death,

$$
u_{L}\left(c_{L}\right)>u_{D}\left(c_{D}\right)
$$

Figure 1 illustrates the insurance choice of three individuals with the same endowment in the two states of the world (represented by point E), and the budget sets, depending on the characteristics of the markets. Individual 2 will neither buy annuities nor life insurance at the respective prices $p_{L}$ and $1-p_{D}$. With perfect markets, she would increase her bequest by taking out a life insurance contract and choose point $\mathrm{G}_{2}$. Individual 1 demands life insurance with imperfect markets $\left(\mathrm{F}_{1}\right)$ and would also opt for a point along the budget line for fair insurance $\left(\mathrm{G}_{1}\right)$. Finally, individual 3 decides for an annuity contract when the markets are imperfect $\left(\mathrm{F}_{3}\right)$ and would not change to life insurance when the insurance markets were perfect $\left(\mathrm{G}_{3}\right)$.

Since the transition from an imperfect to a perfect market can be interpreted as a change in the price of insurance, figure 1 also illustrates the effect of a price change on the demand for annuities and life insurance, respectively. Individual 1 experiences a decrease in the relative price of $c_{D}$ : the positive substitution effect is reinforced by the income effect stemming from the cut in the price $1-p_{D}$ of her life insurance contract so that $\partial c_{D} / \partial p_{D}$ is unambiguously positive. Individual 3 faces a decrease in the price of her asset, and thus both the substitution effect and the income effect lower her demand for survival consumption, i.e., $\partial c_{L} / \partial p_{L}<0 .^{5}$

To derive the marginal rate of substitution between survival and wealth we first differentiate the budget constraint with respect to survival probability, and obtain

$$
\frac{\partial c_{D}}{\partial \pi}=\frac{p_{j}^{\prime}}{p_{j} \cdot\left(1-p_{j}\right)} \cdot\left(c_{D}-V\right)-\frac{p_{j}}{1-p_{j}} \cdot \frac{\partial c_{L}}{\partial \pi} .
$$

Using the envelope theorem we find for the bequest model $(b)$ after some modifications:

$$
m_{b}(\pi, V, H)=-\left.\frac{d V}{d \pi}\right|_{d E U=0}=\frac{p_{j}}{\pi} \cdot \frac{u_{L}-u_{D}}{u_{L}^{\prime}}+\frac{p_{j}^{\prime}}{p_{j}} \cdot\left(c_{D}-V\right) .
$$




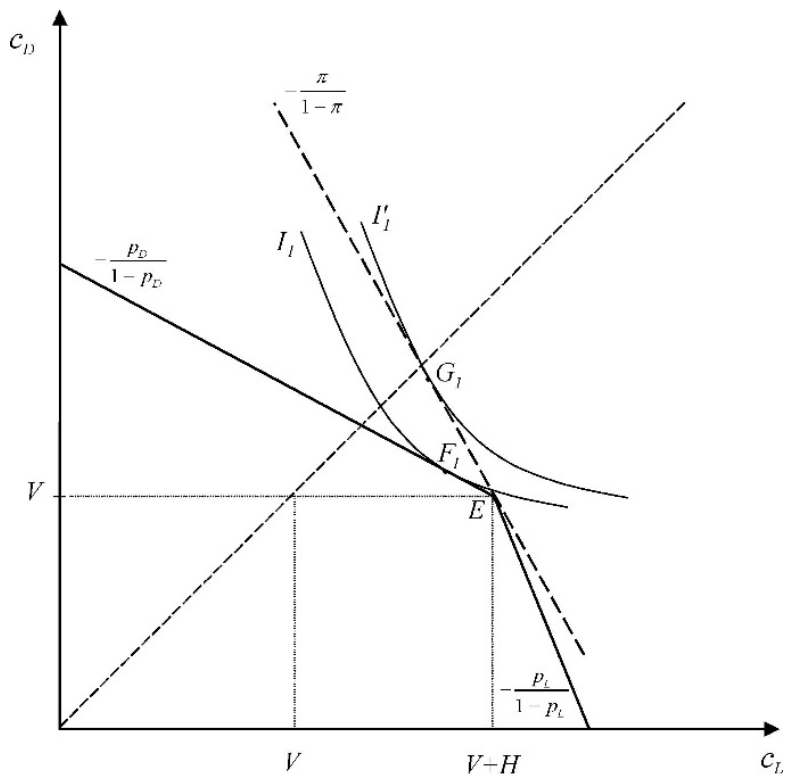

Panel a) Individual 1, buying life insurance

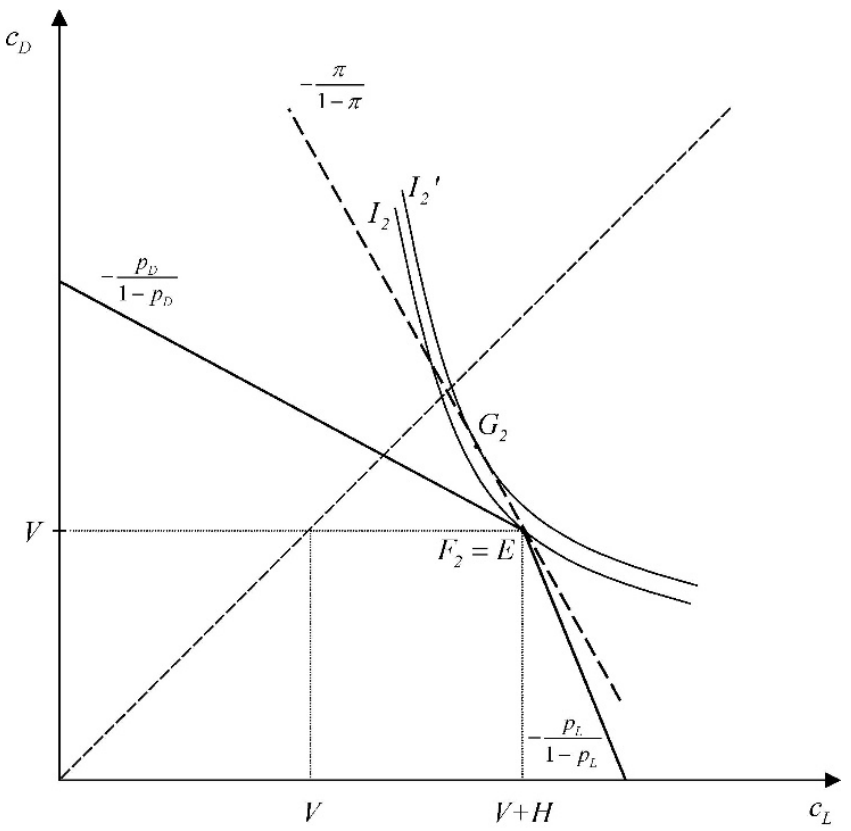

Panel b) Individual 2, not active in the insurance market

Figure 1. Choosing between annuities and life insurance depending on the net-position in the no-insurance case $(F)$. (Continued on next page.) 


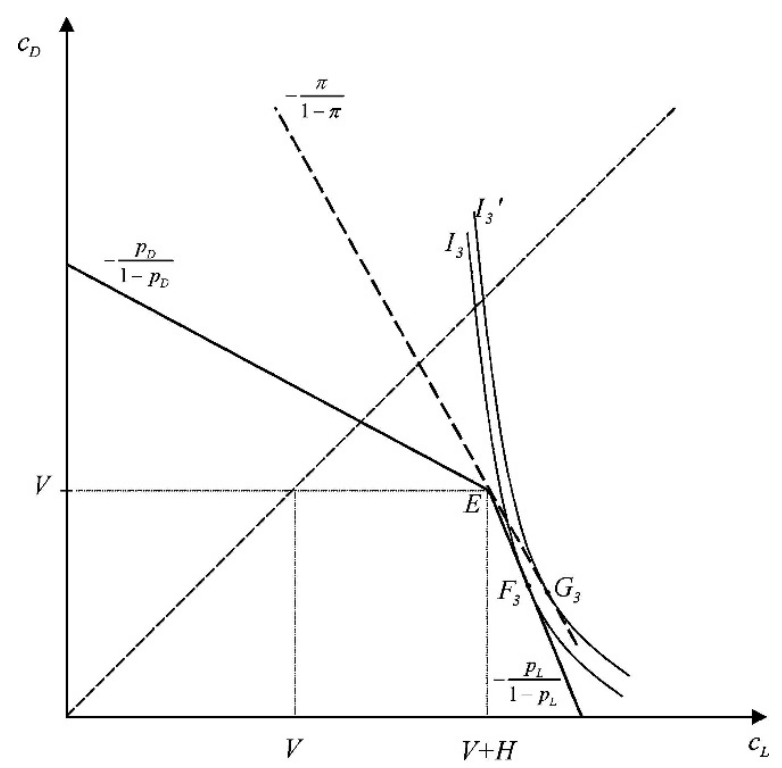

Panel c) Individual 3, buying an annuity contract

Figure 1. (Continued).

Note the similarity between (20) and (4). When bequests are added to the pure consumption model, the value of a statistical life is driven by the utility difference in monetary terms and the difference between optimal bequest and bequeathable wealth. The two parts of the value of a statistical life remain: the direct effect on the expected utility from a change in survival probability and the wealth effect through the insurance market. From (20) we find

$$
\begin{aligned}
\frac{\partial m_{b}}{\partial \pi}= & \frac{p_{j}^{\prime} \cdot \pi-p_{j}}{\pi^{2}} \cdot \frac{u_{L}-u_{D}}{u_{L}^{\prime}}+\frac{p_{j}}{\pi} \cdot \frac{\partial\left(\left(u_{L}-u_{D}\right) / u_{L}^{\prime}\right)}{\partial \pi} \\
& +\frac{p_{j}^{\prime \prime} \cdot p_{j}-p_{j}^{\prime 2}}{p_{j}^{2}} \cdot\left(c_{D}-V\right)+\frac{p_{j}^{\prime}}{p_{j}} \cdot \frac{\partial c_{D}}{\partial \pi}
\end{aligned}
$$

Since (from (19))

$$
\begin{aligned}
\frac{\partial\left(\frac{u_{L}-u_{D}}{u_{L}^{\prime}}\right)}{\partial \pi} & =\frac{\left(u_{L}^{\prime} \cdot \frac{\partial c_{L}}{\partial \pi}-u_{D}^{\prime} \cdot \frac{\partial c_{D}}{\partial \pi}\right) \cdot u_{L}^{\prime}-u_{L}^{\prime \prime} \cdot \frac{\partial c_{L}}{\partial \pi} \cdot\left(u_{L}-u_{D}\right)}{u_{L}^{\prime 2}} \\
& =\frac{\partial c_{L}}{\partial \pi} \cdot\left[1-\frac{\left(u_{L}-u_{D}\right) \cdot u_{L}^{\prime \prime}}{u_{L}^{\prime 2}}\right]-\frac{\partial c_{D}}{\partial \pi} \cdot \frac{\pi}{1-\pi} \cdot \frac{1-p_{j}}{p_{j}} \\
& =\frac{\partial c_{L}}{\partial \pi} \cdot\left[\frac{1}{1-\pi}-\frac{\left(u_{L}-u_{D}\right) \cdot u_{L}^{\prime \prime}}{u_{L}^{\prime 2}}\right]-\frac{p_{j}^{\prime}}{p_{j}^{2}} \cdot \frac{\pi}{1-\pi} \cdot\left(c_{D}-V\right)
\end{aligned}
$$


a combination of (21) and (22) yields

$$
\begin{aligned}
\frac{\partial m_{b}}{\partial \pi}= & -\frac{1}{\pi} \cdot\left(\frac{p_{j}}{\pi}-p_{j}^{\prime}\right) \cdot \frac{u_{L}-u_{D}}{u_{L}^{\prime}}+\frac{\partial c_{L}}{\partial \pi} \cdot \frac{p_{j}}{\pi} \\
& \cdot\left[\frac{1}{1-\pi}-\frac{\pi}{p_{j}} \cdot \frac{p_{j}^{\prime}}{1-p_{j}}-\frac{\left(u_{L}-u_{D}\right) \cdot u_{L}^{\prime \prime}}{u_{L}^{\prime 2}}\right] \\
& +\left[p_{j}^{\prime \prime}+\frac{p_{j}^{\prime 2}}{1-p_{j}}-\frac{p_{j}^{\prime}}{1-\pi}\right] \cdot \frac{\left(c_{D}-V\right)}{p_{j}} .
\end{aligned}
$$

From a derivation of the FOC (16) we find (see the Appendix)

$$
\frac{\partial c_{L}}{\partial \pi}=\frac{-\frac{1-p_{j}}{\pi \cdot(1-\pi)}+\frac{p_{j}^{\prime}}{p_{j}} \cdot\left[1+\frac{u_{D}^{\prime \prime}}{u_{D}^{\prime}} \cdot\left(c_{D}-V\right)\right]}{\frac{u_{L}^{\prime \prime} \cdot\left(1-p_{j}\right)}{u_{L}^{\prime}}+\frac{u_{D}^{\prime \prime} \cdot p_{j}}{u_{D}^{\prime}}}
$$

With risk aversion, even if the individual buys annuities $\left(c_{D}<V\right)$, and thus $\partial c_{L} / \partial \pi$ is likely negative, this does not guarantee that $\partial m_{b} / \partial \pi<0$ since the expression in the second brackets in (23) is not necessarily negative.

The effect on WTP for survival can not be signed either if the prices on the insurance markets do not adjust to a change in survival probability. In this case $\partial c_{L} / \partial \pi>0$, the income effect in (23) disappears, while the second term in (23) is positive. Thus, we have two counteracting effects, leaving the sign of $\partial m_{b} / \partial \pi$ indeterminate.

If we assume a constant loading factor on the fair premium (see (6)), (23) and (24) simplify to

$$
\frac{\partial m_{b}}{\partial \pi}=\frac{\partial c_{L}}{\partial \pi} \cdot(1+\lambda) \cdot\left[\frac{-\lambda \cdot \pi}{a \cdot(1-\pi)}-\frac{\left(u_{L}-u_{D}\right) \cdot u_{L}^{\prime \prime}}{u_{L}^{\prime 2}}\right]+\frac{\lambda}{a \cdot(1-\pi)} \cdot\left(c_{D}-V\right),
$$

where $a \equiv 1-(1+\lambda) \cdot \pi>0,{ }^{6}$ and

$$
\frac{\partial c_{L}}{\partial \pi}=\frac{\frac{\lambda}{1-\pi}+\frac{u_{D}^{\prime \prime}}{\pi \cdot u_{D}^{\prime}} \cdot\left(c_{D}-V\right)}{\frac{a \cdot u_{L}^{\prime \prime}}{u_{L}^{\prime}}+\frac{(1+\lambda) \cdot u_{D}^{\prime \prime}}{u_{D}^{\prime}}}
$$

An increase in survival probability has a (negative) substitution effect on survival consumption as its price rises. The sign of the income effect depends on the individual's position on the insurance market. If the individual demands life insurance $\left(c_{D}>V\right)$, there is a positive income effect from an increase in survival probability, so that the sign of the total effect is unclear. When the individual buys annuities $\left(c_{D}<V\right)$, both the substitution and income effect are negative, and survival consumption decreases. But also in the case with constant loading, the total effect of a change in survival probability on WTP for survival is not clear, so we turn to the two polar cases with perfect and non-existent insurance markets. 


\subsection{Perfect insurance markets}

In the perfect market case ( $p i)$, the value of a statistical life is:

$$
m_{b, p i}(\pi, V, H)=\frac{u_{L}-u_{D}}{u_{L}^{\prime}}+\left(c_{D}-V\right) .
$$

For the marginal change we obtain from (25) for $\lambda=0$

$$
\frac{\partial m_{b, p i}(\pi, V, H)}{\partial \pi}=-\frac{\partial c_{L}}{\partial \pi} \cdot \frac{\left(u_{L}-u_{D}\right) \cdot u_{L}^{\prime \prime}}{u_{L}^{\prime 2}} .
$$

It follows then from (24) that for the perfect insurance case:

$$
\operatorname{sign}\left(\partial m_{b, p i} / \partial \pi\right)=\operatorname{sign}\left(c_{D}-V\right) .
$$

With zero survival income $(H=0), c_{L}>0$ implies $c_{D}<V$ and thus guarantees the WTP to be decreasing in the survival probability. If $H>0$, the sign of $\partial m_{b} / \partial \pi$ is indeterminate and the effect may be reversed. Provided that at the initial equilibrium $\left(c_{L}, c_{D}\right)$, the individual wants to leave more than her bequeathable wealth $V$ to her heirs by converting some of her survival income into life insurance, then, a decrease in the survival probability $\pi$ will make her poorer and thus induce her to lower consumption in case of survival. This in turn will raise marginal utility of wealth in both states of nature and thus depress WTP for survival.

\subsection{No insurance markets}

In the bequest model with non-existent insurance markets $(n i)$, the budget constraints in the two states are

$$
c_{L}=V+H \text { and } c_{D}=V .
$$

Marginal willingness to pay for survival is then

$$
m_{b, n i}(\pi, V, H)=\frac{u_{L}(V+H)-u_{D}(V)}{E U^{\prime}},
$$

with $E U^{\prime}=\pi \cdot u_{L}^{\prime}+(1-\pi) \cdot u_{D}^{\prime}$ as the expected marginal utility of wealth. Here, there is no income effect from a change in survival probability. However, as marginal utilities of wealth can not be equalized across states of the world with missing contingent claims markets, the second derivative may bear a surprise:

$$
\frac{\partial m_{b, n i}(\pi, V, H)}{\partial \pi}=-\left(u_{L}-u_{D}\right) \cdot \frac{u_{L}^{\prime}(V+H)-u_{D}^{\prime}(V)}{\left(E U^{\prime}\right)^{2}} .
$$


The RHS of (32) can not be signed because the sign of the numerator is indeterminate for positive values of survival income. The intuition for the case of a positive sign of $\partial m_{n i, b} / \partial \pi$ is the following: If

$$
u_{L}^{\prime}(V+H)<u_{D}^{\prime}(V)
$$

the marginal utility of wealth upon death exceeds the one upon survival because survival income is lost and bequeathable wealth can not be shifted to the state of death due to the absence of a life insurance market. An increase in the risk of death therefore raises the expected marginal utility of wealth and thus decreases marginal WTP for survival-the opposite of the DAE. This effect may be called "constrained-bequest effect" ${ }^{\text {"7 }}$ because it derives from the inability of the individual to leave as much to her heirs as she desires, which renders the marginal utility of a dollar upon death to exceed the marginal utility of a dollar upon survival.

\section{Conclusion}

In this study, we have re-examined the claim made by Pratt and Zeckhauser [1996] that the value of a statistical life measured by marginal WTP for survival increases with the level of initial mortality risk due to a "dead-anyway effect". We have revisited this hypothesis in models with and without bequests and under different assumption on the characteristics of the insurance markets. Table 1 summarizes the results regarding perfect, imperfect and non-existent insurance markets.

The insights which emerge from the theoretical analysis are as follows:

In general, the reaction of WTP to increases in the survival probability can depend upon three different effects:

(i) Pratt and Zeckhauser's dead-anyway effect which derives from the assumption that marginal utility of a dollar upon death is smaller than the marginal utility of a dollar in the case of survival,

(ii) a "constrained-bequest" effect stating that in the presence of both a bequest motive and human capital and other assets which are lost upon death, the marginal utilities in the two states of the world may behave the opposite way,

(iii) an income effect which says that an increase in survival probability makes the individual either poorer (if she is a net buyer of annuities) or richer (if she buys life insurance) and thereby changes marginal utility of wealth in both states of the world if the usual risk aversion is assumed.

In particular, for individuals without a bequest motive the value of a statistical life always increases with the level of risk exposure: With perfect contingent-claims markets, this result is due to the income effect combined with risk aversion with respect to wealth: an increasing risk makes the individual poorer and thereby raises marginal utility of wealth. Otherwise, it is due to the dead-anyway effect. In this case no assumption about the curvature of the utility is required. 


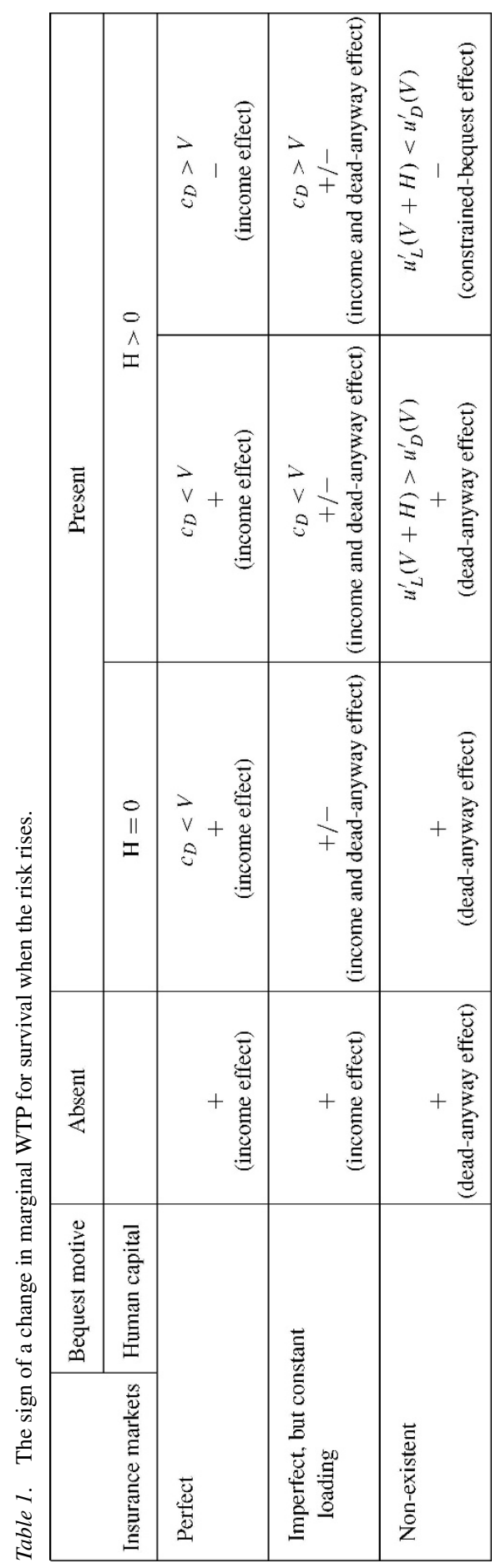


When a bequest motive exists, a reversal of the effect of risk level on WTP for survival may occur-again, independent of the existence of perfect insurance markets. A sufficient condition for this to happen is that a significant portion of total wealth depends upon survival (like human capital) and is lost when the individual dies so that the constrained bequest effect is operative. As a consequence, the necessary correction of survey results for differences in the level of risk exposure may have the opposite direction than proposed by Pratt and Zeckhauser.

The theoretical examination performed in this paper can also help to explain why the empirical evidence on the relation between WTP and the level of risk exposure is so inconclusive. ${ }^{8}$ For instance, Smith and Desvousges [1987] found in their questionnaire study that "in the majority of cases ... the bids are larger at smaller baseline risks" (ibid., p. 99). This finding is compatible with the "constrained bequest effect" although we can not claim that the latter is indeed the explanation for these results. Similarly, Breyer and Grabka [2001] analyzing data from a large panel study, find no significant effect of risk measures such as age and recent hospitalizations on the marginal utility of wealth, thus indirectly casting doubt on the presence of a "dead-anyway" effect.

One of the reasons why the "dead-anyway" effect can not be detected in empirical studies is the presence of extensive pay-as-you-go and funded social old-age insurance. These schemes increase non-bequeathable wealth and increase the likelihood that, given a degree of imperfection in the life insurance market, the marginal utility of a dollar at survival exceeds the one at death.

\section{Appendix: Deriving (24)}

A derivation of the FOC (16) in the form

$$
u_{L}^{\prime} \cdot \pi \cdot\left(1-p_{j}\right)=u_{D}^{\prime} \cdot(1-\pi) \cdot p_{j}
$$

gives

$$
\begin{aligned}
u_{L}^{\prime \prime} & \cdot \frac{\partial c_{L}}{\partial \pi} \cdot \pi \cdot\left(1-p_{j}\right)+u_{L}^{\prime} \cdot\left(1-p_{j}-p_{j}^{\prime} \cdot \pi\right) \\
& =u_{D}^{\prime \prime} \cdot \frac{\partial c_{D}}{\partial \pi} \cdot(1-\pi) \cdot p_{j}+u_{D}^{\prime} \cdot\left(p_{j}^{\prime} \cdot(1-\pi)-p_{j}\right)
\end{aligned}
$$

Collecting the second derivatives on the l.h.s., dividing by $(1-\pi) \cdot p \cdot u_{D}^{\prime}$, and employing the FOC yields:

$$
\begin{aligned}
\frac{u_{L}^{\prime \prime}}{u_{L}^{\prime}} \cdot \frac{\partial c_{L}}{\partial \pi}-\frac{u_{D}^{\prime \prime}}{u_{D}^{\prime \prime}} \cdot \frac{\partial c_{D}}{\partial \pi} & =\frac{p_{j}^{\prime} \cdot(1-\pi)-p_{j}}{(1-\pi) \cdot p_{j}}-\frac{u_{L}^{\prime}}{u_{D}^{\prime}} \cdot \frac{1-p_{j}-p_{j}^{\prime} \cdot \pi}{(1-\pi) \cdot p_{j}} \\
& =\frac{p_{j}^{\prime}}{\left(1-p_{j}\right) \cdot p_{j}}-\frac{1}{\pi \cdot(1-\pi)}
\end{aligned}
$$


again by the FOC. Using (19) and collecting terms gives

$$
\begin{aligned}
& \frac{\partial c_{L}}{\partial \pi}\left(\frac{u_{L}^{\prime \prime}}{u_{L}^{\prime}}+\frac{u_{D}^{\prime \prime}}{u_{D}^{\prime}} \cdot \frac{p_{j}}{1-p_{j}}\right)-\frac{u_{D}^{\prime \prime}}{u_{D}^{\prime}} \cdot \frac{p_{j}^{\prime}}{\left(1-p_{j}\right) \cdot p_{j}} \cdot\left(c_{D}-V\right) \\
& \quad=\frac{p_{j}^{\prime}}{\left(1-p_{j}\right) \cdot p_{j}}-\frac{1}{\pi \cdot(1-\pi)}
\end{aligned}
$$

Multiplying both sides with $\left(1-p_{j}\right)$ and solving for $\partial c_{L} / \partial \pi$ directly leads to equation (24) in the text.

\section{Acknowledgments}

The research for this paper began while the first author was a visiting scholar at the Research School of Social Sciences (Social \& Political Theory Group) of the Australian National University, Canberra. Valuable comments by Ted Bergstrom (UC Santa Barbara), Louis Eeckhoudt (Fucam, University of Mons), Christian Gollier (University of Toulouse) Mathias Kifmann, Normann Lorenz (University of Konstanz), Andreas Werblow (University of Magdeburg), Richard Zeckhauser (Harvard University) and an anonymous referee are gratefully acknowledged.

\section{Notes}

1. While the term "dead-anyway effect" was coined by Pratt and Zeckhauser, the conjecture that WTP rises with the baseline risk goes back at least to Jones-Lee [1974] and Weinstein, Shepard and Pliskin [1980].

2. Pratt and Zeckhauser [1996, p. 750] call this the "realistic case".

3. Since only the share $\pi$ of all participants of the tontine survive, the expected profit of the organizers of the tontine is zero. Fair annuities are very similar to tontine shares.

4. Bergstrom [1982] speaks of a decomposition of the value of life "into a direct or "compensated" effect and a pecuniary effect" (p. 8).

5. Note, however, that the two cross effects $\left(\partial c_{L} / \partial p_{D}\right.$ for individual 1 and $\partial c_{D} / \partial p_{L}$ for individual 3) are indeterminate because substitution and income effects have opposite signs. All these effects will be used below in the derivation of the effects of a change in survival probability on WTP.

6. If $a$ were larger or equal to 1, the individual would not choose an interior optimum.

7. This terminology was suggested by Richard Zeckhauser in private correspondence. Note that Jones-Lee [1974] hints to this case when he writes that $u_{L}^{\prime}(V+H)>u_{D}^{\prime}(V)$ may be violated "when only a very small part of current wealth is bequeathable" (p. 838).

8. For extensive reviews of the empirical literature, see Viscusi [1993] and more recently, Hammitt and Graham [1999].

\section{References}

BERGSTROM, T.C. [1982]: "When is a Man's Life Worth More Than his Human Capital?" in The Value of Life and Safety, Jones-Lee, M.W. (Ed.), North Holland, pp. 3-26.

BREYER, F. and GRABKA, M. [2001]: "Is There a Dead-Anyway Effect in Willingness to Pay for Risk Reduction?" Discussion Paper No. 252, DIW Berlin, http://www.diw.de/english/ publikationen/diskussionspapiere/jahrgang01/. 
FELDER, S., MEIER M., and SCHMITT, H. [2000]: "Health Care Expenditure in the Last Months of Life," Journal of Health Economics, 19(5), 679-695.

JOHANNSON, P.-O. [1996]: "On the Value of a Statistical Life," Journal of Health Economics, 15, 105-113.

JOHANNSON, P.-O. [2002]: "On the Definition and Age-Dependency of the Value of a Statistical Life: a Review and Extension," Journal of Risk and Uncertainty, 25, 251-263.

HAMMITT, J.K. and GRAHAM, J.D. [1999]: "Willingness to Pay for Health Protection: Inadequate Sensitivity to Probability?" Journal of Risk and Uncertainty, 18, 33-62.

JONES-LEE, M.W. [1974]: "The Value of Changes in the Probability of Death or Injury," Journal of Political Economy, 82, 835-849.

JONES-LEE, M.W. [1976]: The Value of Life: An Economic Analysis. Chicago: University Press.

PRATT, J.W. and ZECKHAUSER, R.J. [1996]: "Willingness to Pay and the Distribution of Risk and Wealth," Journal of Political Economy, 104, 747-763.

ROSEN, S. [1988]: "The Value of Changes in Life Expectancy," Journal of Risk and Uncertainty, 1, 285-304.

SMITH, V.K. and DESVOUSGES, W.H. [1987]: "An Empirical Analysis of the Economic Value of Risk Changes," Journal of Political Economy, 95, 89-114.

VISCUSI, W.K. [1993]: "The Value of Risks to Life and Health," Journal of Economic Literature, 31, 1912-1946. WEINSTEIN, M.C., SHEPARD, D.S., and PLISKIN, J.S. [1980]: "The Economic Value of Changing Mortality Probabilities," Quarterly Journal of Economics, 94, 373-396. 